\title{
Detection with Embedded Known Symbols: Optimal Symbol Placement and Equalization
}

\author{
Srihari Adireddy and Lang Tong \\ School of Electrical Engineering \\ Cornell University, Ithaca, NY 14853 \\ \{srihari,ltong\}@ee.cornell.edu
}

\begin{abstract}
The detection of a data sequence with embedded known symbols is considered. For a class of symbol-by-symbol decision feedback receivers, known symbol distributions optimal with respect to the criterion of average mean square error(A-MSE) are presented. Optimal design of the decision feedback receiver is also obtained. Simulation results show that, compared to the performance with conventional symbol placement strategy, considerable gain can be obtained by the joint optimization of symbol placement and equalizer.
\end{abstract}

\section{INTRODUCTION}

In data communications, especially in packet transmission schemes, usually there are known symbols embedded in the data stream. Figure 1 shows a typical data stream. These known symbols serve various purposes such as synchronization, training of receivers and packet identification. For example, in the packet structure of GSM, there are 26 consecutive known symbols in the middle of every packet and 3 known symbols at each end. For transmissions over a high frequency (HF) channel, known symbol sequences as long as the duration of the channel are placed periodically in the data stream.

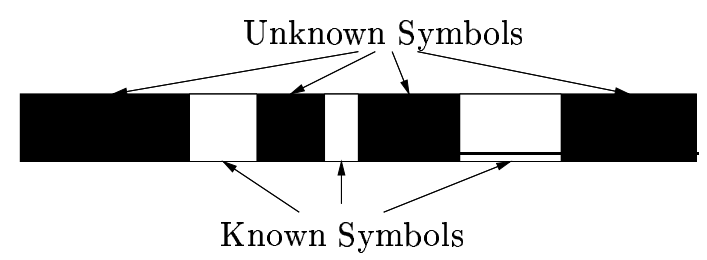

Figure 1: Typical data stream.

\footnotetext{
${ }^{0}$ This work was supported in part by the Philips Research and by the National Science Foundation under Contract CCR-9804019.
}

For a given receiver structure, the placement of known symbols may affect the receiver performance. This raises the following questions: given the percentage of known symbols to be embedded in the data stream,

1. What is the optimal placement of known symbols?

2. Does the placement of known symbols depend on the transmission channel?

3. What is the optimal equalizer?

Previous work in equalizer design in the presence of known symbols has been mainly with respect to iterative equalization $[6,2,1]$. Almost all of them design the optimum MSE equalizer assuming that they have tentative decisions on all the symbols. Mueller and Salz [7] examined the problem of obtaining the optimum MSE forward filter and feedback filters, given that only a subset of data symbols are known. Hsu [4], and more recently, Kaleh [5] have addressed the equalization problem for block transmission systems, where known symbols are placed periodically in the data stream separating the blocks of data. Usually, in this scenario the length of consecutive known symbols is greater than the time spread of the channel. The starting point of all the previous work is the assumption of a fixed distribution of known symbols.

The main objective of this paper is to optimize the symbol placement and equalization jointly. Very little work has been reported in this regard. It turns out that conventional strategy of placing known symbols together in clusters carries a penalty in performance. For some cases, the loss of performance can be substantial. For a particular class of symbol-by-symbol decision feedback structures, we give known symbol placement schemes that are optimal under certain conditions that are easily satisfied. Surprisingly, as shown 
in section 4 , the optimal symbol placement does not depend on the actual channel coefficients. Because the placement of known symbols is a transmitter technique, the proposed strategy is particularly attractive for broadcast applications. A general optimal decision feedback receiver is also presented.

This paper is organized as follows. In Section 2 we give the problem statement and necessary assumptions. In Section 3 we consider first the optimization of feedback structure. Here we present a design that is optimal for any reasonable criterion (in the sense of Ericson [3]). In Section 4 we present the optimal symbol placement followed by, in Section 5 the optimal forward filter. Finally a simulation example is used to demonstrate the potential gain in performance when optimal symbol placement is used in place of the conventional clustered symbol placement.

\section{PROBLEM STATEMENT}

\subsection{The Model}

We consider the transmission of data sequence $s_{k}$ over a linear ISI channel with additive white Gaussian noise of variance $\sigma_{n}^{2}$ as shown in Figure 2. The channel is assumed to have a finite impulse response of order $L$. The input sequence, as illustrated in Figure 1, consists of $P$ known symbols and $N$ unknown symbols although our results generalize naturally to the case when the number of unknown symbols goes to infinity with a fixed redundancy ratio $P / N$. Outside the duration of transmission, the input sequence is assumed to be zero. We further assume that unknown symbols are independent and identically distributed with zero mean and variance $\sigma_{s}^{2}$.

We consider general symbol-by-symbol decision feedback receivers with the structure shown in Figure 2 where a linear time invariant filter $f(z)$ of order $L_{f}$ is followed by a general decision feedback detector $B$. The constraint that the forward filter is time invariant greatly simplifies the implementation. We do not impose any constraints on the feedback structure as of now except that it has access to all the known symbols in the data stream and all the past decisions. We assume that the equalizer has a detection delay of $d$. Past decisions are assumed to be correct in the analysis.

\subsection{The Performance Criterion}

Once we assume that the input data stream is composed of known and unknown symbols, the input sequence is no longer stationary. The performance of an

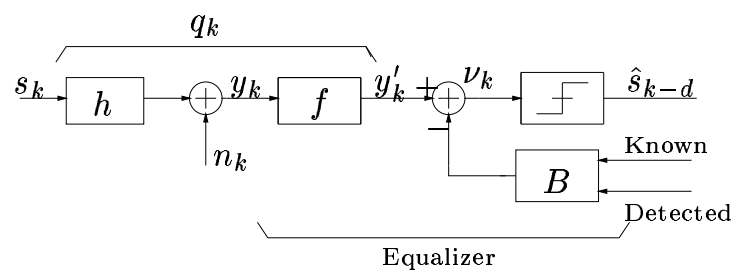

Figure 2: Equalizer Structure

equalizer for such a stream depends in general on the position of the symbol being detected, and hence the widely used mean square error (MSE) criterion needs to be modified to take into account the performance at all the positions in the stream. In this paper, we consider the average mean square error (A-MSE) defined as

$$
\mathcal{M}_{a}(\mathcal{P}, f, B)=\frac{1}{N} \sum_{(k-d) \notin \mathcal{P}} \mathrm{E}\left(v_{k}-s_{k-d}\right)^{2},
$$

where $\mathcal{P}$ is the index set for the known symbols. Our objective to perform the joint optimization of A-MSE

$$
\min _{\mathcal{P}, f, B} \mathcal{M}_{a}(\mathcal{P}, f, B)
$$

\section{OPTIMAL FEEDBACK STRUCTURES}

We begin the joint optimization of A-MSE with the design of the optimal feedback filter after fixing the known symbol distribution $\mathcal{P}$ and forward filter $f$. Our result here is more general in the sense that the resulting feedback structure is optimal for any reasonable performance criterion. As defined by Ericson [3], a reasonable criterion is one that becomes worse with any independent additive disturbance in the observation.

Lemma 1 For a given linear time invariant filter $f$ and known symbols placement $\mathcal{P}$, the feedback structure $B$ which subtracts the contribution of all the known and detected symbols to ISI in $y_{k}^{\prime}$ is optimal for any reasonable performance criterion.

Figure 3 illustrates a symbol-by-symbol decision feedback equalizer receiver that can be made optimal with respect to the feedback structure by suitable choice of the feedback filters $b$ and $c$. This structure is different from the conventional DFE in that two feedback filters are used. Filter $b$ is a causal (with reference to the detected symbol) linear filter that subtracts all interference from the previously detected symbols (postcursor ISI). Filter $c$ subtracts all precursor ISI due to 
known symbols. For this reason, the DFE shown in Figure 3 is referred to as the Precursor Cancellation DFE (PC-DFE).

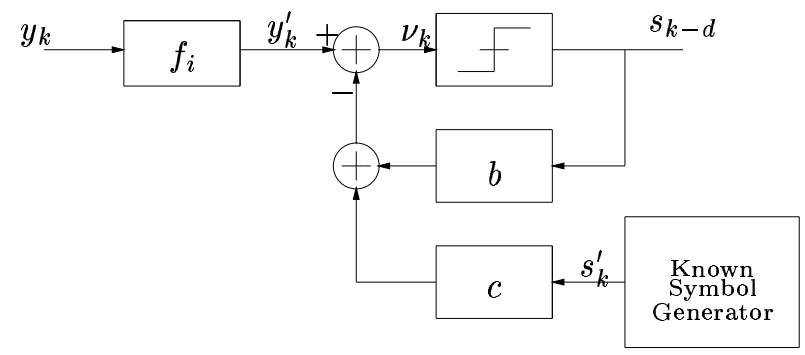

Figure 3: Precursor Cancellation DFE

The condition on $L_{b}$ (number of taps in filter $b$ ) and $L_{c}$ (number of taps in filter $c$ ) so that the structure shown is optimal is

$$
L_{b} \geq L+L_{f}-d \quad L_{c} \geq d
$$

Provided the above condition is satisfied, the optimal choice of taps is

$$
\begin{aligned}
b_{i} & =\left\{\begin{array}{cl}
q_{i+d} & i=1, \cdots, L+L_{f}-d \\
0 & \text { otherwise }
\end{array}\right. \\
c_{i} & = \begin{cases}q_{i} & i=0, \cdots, d-1 \\
0 & \text { otherwise }\end{cases}
\end{aligned}
$$

Structures conceptually similar to PC-DFE have been the starting point for previous work in equalization with known future symbols [2, 7]. Lemma 1 mandates that the choice of the feedback structure of $\mathrm{PC}-\mathrm{DFE}$ is in fact optimal for any reasonable criterion.

\section{OPTIMAL SYMBOL PLACEMENT}

We now optimize the distribution of the known symbols for the optimally designed receiver. The optimal receiver is a function of the propagation channel and intuitively we would also expect the optimal known symbol distribution to be a function of the channel. But that would render the optimization of symbol placement useless for broadcast applications. Fortunately, the following theorem shows that the optimal symbol distribution is independent of the channel.

Theorem 1 For a fixed decision delay $d$ and a given $f$, if $\frac{P}{N+P} \leq \frac{1}{d+1}-\frac{d+\left(L_{f}-2 d\right)^{+}}{(N+P)(d+1)}$ (where $x^{+}$denotes the non-negative part of $x$ ) a symbol distribution $\mathcal{P}$ is optimal if and only if

$$
\begin{gathered}
\left|x_{i}-x_{j}\right|>d, \forall x_{i}, x_{j} \in \mathcal{P} \text { and } i \neq j . \\
x_{1} \geq \max \left(d, L_{f}-d\right) \text { and } x_{P} \leq N+P-d-1
\end{gathered}
$$

If $N$ is large enough to ignore the end effects and if the percentage of known symbols is less than $\frac{100}{(d+1)} \%$, then a distribution $\mathcal{P}$ is optimal if and only if

$$
\left|x_{i}-x_{j}\right|>d, \forall x_{i}, x_{j} \in \mathcal{P} \text { and } i \neq j .
$$

Conventionally, known symbols are placed in clusters. Theorem 1 suggests that symbol detections can be improved if known symbols are distributed across the data stream. Figure 4 gives one example of a distribution that is optimal. The optimal symbol dis-

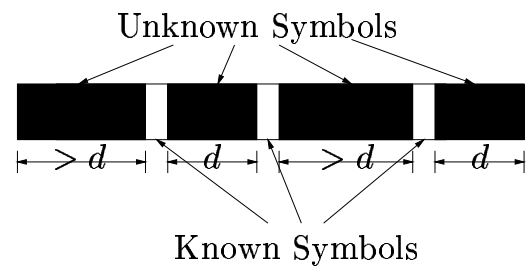

Figure 4: Example of a distribution that is Optimal

tribution is independent of the propagation channel. This property is crucial in broadcast applications. Also, the optimal symbol distribution is not unique; many strategies are equivalent in terms of their A-MSE performance. The theorem does not say anything about the optimal placement schemes, if the percentage of known symbols is greater than $\frac{100}{(d+1)} \%$. We conjecture that in this case there is no distribution which is uniformly best for all channels. But even in this case, we would expect distributing the known symbols across the data stream to be better than clustering.

\section{OPTIMAL FORWARD FILTER}

In this section we complete the joint optimization of the A-MSE. We assume that the total number of known symbols $P$ is such that optimal distributions as indicated in the previous section are possible. Also we assume that the number of unknown data symbols $N$ is large enough to ignore the end effects. The optimal forward filter assuming the feedback structure and the placement are chosen optimally is given by

$$
\mathbf{f}^{o}=\left(\mathbf{H}_{d}^{H} \mathbf{\Lambda} \mathbf{H}_{d}+\lambda \mathbf{I}_{L_{f}+1}\right)^{-1} \mathbf{H}_{d}^{H} \mathbf{e}_{d}
$$

where

$$
\mathbf{H}_{d}=\left[\begin{array}{cccc}
h_{0} & 0 & \ldots & 0 \\
h_{1} & h_{0} & \ldots & 0 \\
\vdots & \vdots & \ddots & \\
& & & \\
h_{d} & h_{d-1} & \ldots & h_{d-L_{f}}
\end{array}\right]
$$


and $\boldsymbol{\Lambda}=\operatorname{diag}\left(1-\frac{P}{N}, \ldots, 1-\frac{P}{N}, 1\right)$ The vector $\mathbf{e}_{d}$ is a unit vector of length $d+1$ whose last element is equal to 1 and the rest are equal to zeros and $\lambda$ is equal to $\frac{\sigma_{n}^{2}}{\sigma_{s}^{2}}$. The minimum A-MSE obtained by the joint optimization with respect to $f, B, \mathcal{P}$ is given by

$$
\mathcal{M}\left(\mathbf{f}^{o}\right)=\mathbf{e}_{d}^{H}\left(\boldsymbol{\Lambda}^{-1}+\frac{1}{\lambda} \mathbf{H}_{d} \mathbf{H}_{d}^{H}\right)^{-1} \mathbf{e}_{d}
$$

When $P=0$, the optimum forward filter turns out to be the same as the one for conventional DFE. As $P$ increases, the power in the precursors of optimum $q_{i}$ increases because known symbols can be used to cancel the precursor ISI.

\section{SIMULATIONS}

We tested the joint optimization strategy by comparing the performance of the MMSE-DFE with known symbol placement as in the GSM-standard with that of PC-DFE with the proposed known symbol placement scheme. The packet structure of a normal burst in GSM was used for the simulation. The data structure within a normal burst consists of 148 bits out of which 116 are information bearing bits. There is a 26 bit training sequence in the middle of the packet. Also there are 3 start bit and 3 stop bits present in the packet.

The channel used for the simulation was $h=[0.407$ $0.8150 .407]$. The order of the forward filter $L_{f}$ was equal to 1 . The detection delay $d$ was set at 1 . The feedback filters of the conventional DFE and the PCDFE were chosen to be as long as required for complete ISI cancellation. Figure 5 gives the performance of MMSE-DFE for the GSM packet, the performance of PC-DFE if the known symbols were distributed as suggested by Theorem 1 and also the performance of PC-DFE if the packet had $50 \%$ known symbols that were distributed optimally.

This simulation shows the potential gain present in the joint optimization of the equalizer and placement schemes. We expect the performance gain to increase with increasing SNR since then the performance loss in a conventional DFE is mainly due to the precursor ISI. Also on channels for which the power of precursors is large, we expect a high performance gain.

\section{CONCLUSIONS}

The known symbols present in the data stream can aid in equalization. Decision feedback receivers that use the known symbols for detection have been introduced. The performance of the equalizer depends

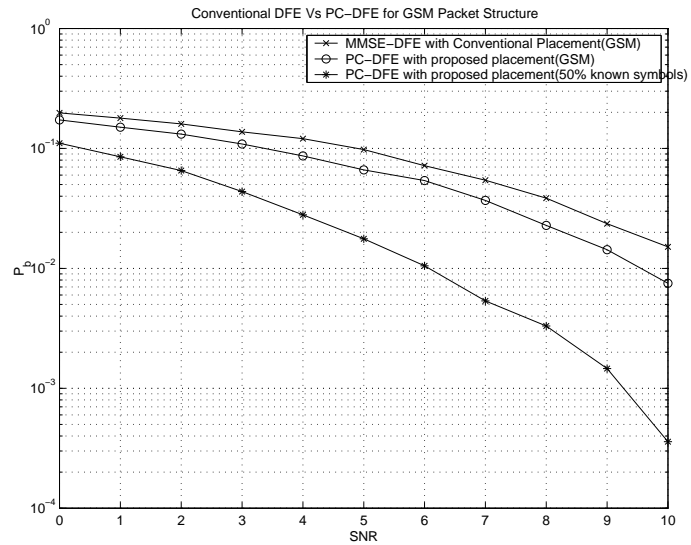

Figure 5: PC-DFE with Proposed Known Symbol Known Symbol Distribution given by the GSM Standard

in general on the forward filter, feedback structure and the positions of the known symbols. The A-MSE criterion which takes into account the performance of the equalizer at all positions has been jointly optimized. The optimal feedback structure,placement strategy and forward filter have been obtained for a few cases. Simulations indicate that there is potential performance gain from the joint optimization.

\section{REFERENCES}

[1] A.Gersho and T.L.Lim. "Adaptive Cancellation of Intersymbol Interference for Data Transmission". Bell System Technical Journal, 60(11):1997-2021, November 1981.

[2] D.T.M.Slock and E.D.Carvalho. "Burst Mode NonCausal Decision-Feedback Equalization and Blind MLSE". Proceedings of IEEE GLOBECOM, pages 4650, November 1996.

[3] T. Ericson. "Structure of Optimum Receiving Filters in Data Transmission Systems". IEEE Trans. Information Theory, pages 352-353, May 1971.

[4] F.M.Hsu. "Data directed estimation techniques for single-tone HF modems' '. In Proc. IEEE MILCOM'85, pages 12.4.1-12.4.10, 1985.

[5] G.K.Kaleh. "Channel equalization for block transmission systems". IEEE J. Sel. Areas Comms., 13(1):110121, January 1995.

[6] J.G.Proakis. "Adaptive Non Linear Filtering Techniques for Data Transmission". Proceedings of the IEEE Symposium on Adaptive Processes, pages XV.2.1-5, 1970.

[7] M.S.Mueller and J.Salz. "A Unified Theory for Data Aided Equalization". Bell System Technical Journal, 60(11):2023-2038, November 1981. 